\title{
THE PRESSURE FUNCTION FOR PRODUCTS OF NON-NEGATIVE MATRICES
}

\author{
De-Jun Feng And Ka-Sing LaU
}

\begin{abstract}
Let $\left(\Sigma_{A}, \sigma\right)$ be a subshift of finite type and let $M(x)$ be a continuous function on $\Sigma_{A}$ taking values in the set of non-negative matrices. We extend the classical scalar pressure function to this new setting and prove the existence of the Gibbs measure and the differentiability of the pressure function. We are especially interested on the case where $M(x)$ takes finite values $M_{1}, \cdots, M_{m}$. The pressure function reduces to $P(q):=\lim _{n \rightarrow \infty} \frac{1}{n} \log \sum_{J \in \Sigma_{A, n}}\left\|M_{J}\right\|^{q}$. The expression is important when we consider the multifractal formalism for certain iterated function systems with overlaps.
\end{abstract}

\section{Introduction}

Let $\sigma$ be the shift map on $\Sigma=\{1,2, \cdots, m\}^{\mathbb{N}}, m \geq 2$. As usual $\Sigma$ is endowed with the metric $d(x, y)=m^{-n}$ where $x=\left(x_{k}\right), y=\left(y_{k}\right)$ and $n$ is the smallest of the $k$ such that $x_{k} \neq y_{k}$. Given an $m \times m$ matrix $A$ with entries 0 or 1 , we consider the subshift of finite type $\left(\Sigma_{A}, \sigma\right)$ (see $\left.[\mathrm{B}]\right)$. We shall always assume that $A$ is primitive.

Suppose $M$ is a continuous function on $\Sigma_{A}$ taking values in the set of all non-negative $d \times d$ matrices. For $q \in \mathbb{R}$, we define the pressure function $P(q)$ of $M$ by

$$
P(q)=\lim _{n \rightarrow \infty} \frac{1}{n} \log \sum_{J \in \Sigma_{A, n}} \sup _{x \in[J]}\left\|M(x) M(\sigma x) \ldots M\left(\sigma^{n-1} x\right)\right\|^{q},
$$

where $\Sigma_{A, n}$ denotes the set of all admissible indices of length $n$ over $\{1, \ldots, m\}$; for $J=j_{1} \cdots j_{n} \in \Sigma_{A, n},[J]$ denotes the cylinder set $\left\{x=\left(x_{i}\right) \in \Sigma_{A}: x_{i}=\right.$ $\left.j_{i}, 1 \leq i \leq n\right\},\|\cdot\|$ denotes the matrix norm defined by $\|B\|:=\mathbf{1}^{t} B \mathbf{1}, \mathbf{1}^{t}=$ $(1,1, \ldots, 1)$. By using a subadditive argument, it is easy to show that for $q>0$, the limit in the above definition exists. With some additional conditions on the matrices (see Theorem 1.1), the limit exists for $q \in \mathbb{R}$.

The pressure function of the scalar case (i.e., $M(x)=e^{\phi(x)}$ where $\phi(x)$ is a real valued function called the potential of the subshift) has been studied

Received June 9, 2001.

Key words and phrases: Pressure, Product of matrices, Gibbs measures, Iterated function systems, Hausdorff dimension, Multifractal formalism.

The first author was partially supported by a HK RGC grant in Hong Kong and the Special Funds for Major State Basic Research Projects in China. The second author is supported by a HK RGC grant. 
in great detail in statistical mechanics and dynamical systems in conjunction with the Gibbs measure, the entropy and the variational principle (c.f., e.g., $[\mathrm{B}],[\mathrm{P}],[\mathrm{R}])$; it has also been used to study the multifractal structure of the self-similar (or self-conformal) measures generated by iterated function systems (IFS) with no overlap (the open set condition) ([MU],[FL]). By identifying with the symbolic space, such self-similar measure $\mu$ is actually a Gibbs measure and the pressure function is directly related to the scaling spectrum of $\mu$ [FL, Theorem 3.3]. In all the above cases, the pressure functions under consideration are differentiable (actually real analytic). This property is essential to investigate the phase transition in thermodynamics and for the multifractal formalism in the dimension theory of fractals.

In the recent investigation of the self-similar measures generated by iterated function systems with overlaps, it is seen that in many interesting cases, such measure $\mu$ can be put into a vector form with a new non-overlapping IFS and with matrix weights ([LN1,2],[LNR],[Fe1],[FeO]). In this way, the validity of the multifractal formalism depends on the differentiability of the pressure function $P(q)$ in (1.1) (more precisely (1.4) in the following) [LN2]. In another direction, the expression of the matrix product in (1.1) also appears in the study of the scaling functions in wavelet theory (the matrices are allowed to have negative entries) in the form of $L^{q}$-joint spectral radius and the $L^{q}$-Lipschitz exponent ([DL1, 2], [LM] ); the problem of differentiability of the $P(q)$ also appears there. So far there is no general theorem to guarantee this fact other than some special cases (e.g., [LN1],[FLN],[Fe1],[FeO],[DL2]).

The main purpose of this paper is to consider the pressure functions and the Gibbs measures for the products of matrices. We first study the case that the matrices $M(x), x \in \Sigma_{A}$ are positive, we prove the following fundamental theorems.

Theorem 1.1. Suppose that $M$ is a Hölder continuous function on $\Sigma_{A}$ taking values in the set of positive $d \times d$ matrices. Then for any $q \in \mathbb{R}$, there is a unique $\sigma$-invariant, ergodic probability measure $\mu_{q}$ on $\Sigma_{A}$ of which one can find constants $C_{1}>0, C_{2}>0$ such that

$$
C_{1} \leq \frac{\mu_{q}([J])}{\exp (-n P(q)) \cdot\left\|M(x) M(\sigma x) \ldots M\left(\sigma^{n-1} x\right)\right\|^{q}} \leq C_{2}
$$

for any $n>0, J \in \Sigma_{A, n}$ and $x \in[J]$.

The above measure $\mu_{q}$ is called the Gibbs measure associated with $M$ and $q$. We remark that the theorem generalizes the classical existence result of the Gibbs measure for a real-valued $M(x)$ (see [B, §1.4]). The positivity of the matrices is used to yield the follow simple estimate (Lemma 2.1)

$$
\left\|M(x) \cdots M\left(\sigma^{n+\ell-1} x\right)\right\| \approx\left\|M(x) \cdots M\left(\sigma^{n-1} x\right)\right\| \cdot\left\|M\left(\sigma^{n} x\right) \cdots M\left(\sigma^{n+\ell-1} x\right)\right\| .
$$


By using this we can apply a technique of Brown, Michon and Peyrière [BMP] and Carleson $[\mathrm{C}]$ to construct a certain ergodic measure which is the Gibbs measure $\mu_{q}$. The $\mu_{q}$ has the following quasi-Bernoulli property (Heurteaux $[\mathrm{H}]$ ): there exists $C>0$ such that for any $n, k \in \mathbb{N}$ with $I \in \Sigma_{A, n}, J \in \Sigma_{A, k}$ and $I J \in \Sigma_{A, n+k}$

$$
C^{-1} \mu_{q}([I]) \mu_{q}([J]) \leq \mu_{q}([I J]) \leq C \mu_{q}([I]) \mu_{q}([J])
$$

This together with a result of Heurteaux $[\mathrm{H}]$ imply:

Theorem 1.2. Under the condition of Theorem 1.1, $P(q)$ is differentiable for $q \neq 0$.

As an application, we let

$$
E(\alpha):=\left\{x \in \Sigma_{A}: \lim _{n \rightarrow \infty} \frac{\log \| M(x) M(\sigma x) \cdots M\left(\sigma^{n-1}(x) \|\right.}{n}=\alpha\right\} .
$$

We prove the following dimension formula.

Theorem 1.3. Under the same assumption of Theorem 1.1, we have for any $\alpha=P^{\prime}(q), q \neq 0$,

$$
\operatorname{dim}_{H} E(\alpha)=\frac{1}{\log m}(-\alpha q+P(q))
$$

where $\operatorname{dim}_{H}$ denotes the Hausdorff dimension.

The above theorems depend very much on the positivity of the matrix-valued $M(x)$. In order to extend them to non-negative matrix-valued functions, we have to impose more conditions on $M(x)$ :

(H2) $\quad M$ is irreducible in the following sense: there exists $r>0$ such that for any $i, j \in\{1,2, \ldots, m\}$,

$$
\sum_{k=1}^{r} \sum_{K \in \Sigma_{A, k ; i, j}} M_{K}>\mathbf{0}
$$

where $\Sigma_{A, k ; i, j}$ denotes the set of all $K \in \Sigma_{A, k}$ such that $i K j \in \Sigma_{A, k+2}$.

We see that under the assumption (H1), the pressure function in (1.1) can be re-written as

$$
P(q)=\lim _{n \rightarrow \infty} \frac{1}{n} \log \sum_{J \in \Sigma_{A, n}}\left\|M_{J}\right\|^{q} .
$$

where $M_{J}=M_{j_{i}} \cdots M_{j_{n}}$. If $\Sigma_{A}=\Sigma$ is the symbolic space with a full shift, then (H2) is equivalent to $\sum_{k=1}^{r}\left(M_{1}+\cdots+M_{m}\right)^{k}>\mathbf{0}$.

In this new setting, we use (H2) to adjust (1.3) and the required lemmas, the Gibbs measure $\mu_{q}$ is shown to exist for $q>0$. This time $\mu_{q}$ only satisfies $\mu_{q}([I J]) \leq C \mu_{q}([I]) \mu_{q}([J])$ instead of $(1.4)$; nevertheless we can still prove the differentiability of $P(q), q>0$ as in Theorem 1.2. Theorem 1.3 can be adjusted likewise (see Theorem 3.3 and Theorem 3.4). As an application, the first author 
proves the smoothness of the $L^{q}$-spectrum $(q>0)$ and the multifractal formalism for a class of self-similar measures with overlaps (including the Bernoulli convolutions associated with Pisot numbers) in a forthcoming paper [Fe2].

For the organization of the paper, we prove the above results for the positive matrix-valued functions in Section 2. In Section 3, we modify the proofs for the non-negative matrix-valued functions with (H1) and (H2). In Section 4, we give an illustration of reducing an IFS with overlap to a vector-valued IFS with no overlap, and the pressure function in (1.6) arises. We also give some remark on the theorems and raise a few unsettled problems.

\section{Positive Matrices}

In this section we assume that $M$ is a Hölder continuous function on $\Sigma_{A}$ taking values in the set of all positive $d \times d$ matrices.

For any two families of positive numbers $\left\{a_{i}\right\}_{i \in \mathcal{I}},\left\{b_{i}\right\}_{i \in \mathcal{I}}$, we write, for brevity, $a_{i} \approx b_{i}$ to mean the existence of a constant $C>0$ such that $C^{-1} a_{i} \leq$ $b_{i} \leq C a_{i}$ for all $i \in \mathcal{I} ; a_{i} \preccurlyeq b_{i}$ to mean the existence of a constant $C>0$ such that $a_{i} \leq C b_{i}$ for all $i \in \mathcal{I}$ and $a_{i} \succcurlyeq b_{i}$ means $b_{i} \preccurlyeq a_{i}$.

We start with a simple lemma.

Lemma 2.1. For any $x \in \Sigma_{A}, n, \ell \in \mathbb{N}$,

$\left\|M(x) \cdots M\left(\sigma^{n+\ell-1} x\right)\right\| \approx\left\|M(x) \cdots M\left(\sigma^{n-1} x\right)\right\| \cdot\left\|M\left(\sigma^{n} x\right) \cdots M\left(\sigma^{n+\ell-1} x\right)\right\|$

(the involved constant in $\approx$ is independent of $n, \ell$ and $x$ ).

Proof. It is clear that

$$
\left\|M(x) \cdots M\left(\sigma^{n+\ell-1} x\right)\right\| \leq\left\|M(x) \cdots M\left(\sigma^{n-1} x\right)\right\| \cdot\left\|M\left(\sigma^{n} x\right) \cdots M\left(\sigma^{n+\ell-1} x\right)\right\| .
$$

To prove the reverse inequality, we observe that $M$ is positive and continuous, there is a constant $C>0$ such that

$$
\frac{\min _{i, j} M_{i, j}(x)}{\max _{i, j} M_{i, j}(x)} \geq C \quad \forall x \in \Sigma_{A} .
$$

This implies that $M(x) \geq \frac{C}{d} E M(x)\left(A \geq B\right.$ means that $A_{i, j} \geq B_{i, j}$ for each index $(i, j))$ where $E=\left(E_{i, j}\right)_{1 \leq i, j \leq d}$ is the matrix whose entries are all equal to 1 . Let 1 be the $d$-dimensional column vector each coordinate of which is 1 . Then

$$
\begin{aligned}
\left\|M(x) \cdots M\left(\sigma^{n+\ell-1} x\right)\right\| & \geq\left\|M(x) \cdots M\left(\sigma^{n-1} x\right) \frac{C}{d} E M\left(\sigma^{n} x\right) \cdots M\left(\sigma^{n+\ell-1} x\right)\right\| \\
& =\frac{C}{d}\left\|M(x) \cdots M\left(\sigma^{n-1} x\right) \mathbf{1}^{t} \mathbf{1} M\left(\sigma^{n} x\right) \cdots M\left(\sigma^{n+\ell-1} x\right)\right\| \\
& =\frac{C}{d}\left\|M(x) \cdots M\left(\sigma^{n-1} x\right)\right\| \cdot\left\|M\left(\sigma^{n} x\right) \cdots M\left(\sigma^{n+\ell-1} x\right)\right\| .
\end{aligned}
$$


We define

$$
s_{n}(I, q)=\sup _{x \in[I]}\left\|M(x) M(\sigma x) \ldots M\left(\sigma^{n-1} x\right)\right\|^{q} \quad \forall I \in \Sigma_{A, n}
$$

and

$$
s_{n}(q)=\sum_{I \in \Sigma_{A, n}} s_{n}(I, q)
$$

Lemma 2.2. For a fixed $q \in \mathbb{R}$,

$$
s_{n}(I, q) \approx\left\|M(x) M(\sigma x) \ldots M\left(\sigma^{n-1} x\right)\right\|^{q} \quad \forall I \in \Sigma_{A, n}, x \in[I] .
$$

Proof. For any $n \in \mathbb{N}$, define

$$
\eta_{n}=\sup \left\{\frac{M_{i, j}(x)}{M_{i, j}(y)}: I \in \Sigma_{A, n}, x, y \in[I], 1 \leq i, j \leq d\right\} .
$$

Since each $M_{i, j}$ is positive and Hölder continuous, we have $\left|\log \eta_{n}\right| \leq \mathrm{Cm}^{-\alpha n}$ for some $C>0$ and $0<\alpha<1$. It follows easily that $\eta:=\prod_{n=1}^{\infty} \eta_{n}<\infty$ and hence for $x \in[I]$,

$$
\left\|M(x) \ldots M\left(\sigma^{n-1} x\right)\right\|^{q} \leq s_{n}(I, q) \leq \eta^{|q|}\left\|M(x) \ldots M\left(\sigma^{n-1} x\right)\right\|^{q} .
$$

We have assumed that $A$ is primitive, there is an integer $p>0$ such that $A^{p}>\mathbf{0}$. This implies that for any $I \in \Sigma_{A, n}, J \in \Sigma_{A, \ell}$, there exists $K \in \Sigma_{A, p}$ such that $I K J \in \Sigma_{A, n+\ell+p}$.

Lemma 2.3. Let $p$ be such that $A^{p}>\mathbf{0}$. Then for a fixed $q \in \mathbb{R}$,

(i) $s_{\ell}(q) \approx s_{\ell-p}(q)$ for all $\ell>p$;

(ii) For $I \in \Sigma_{A, n}, \ell>p, \sum_{J} s_{n+\ell}(I J, q) \approx \sum_{J} s_{n+\ell}(J I, q) \approx s_{n}(I, q) s_{\ell}(q)$ where the first (second) sum is taken over all $J \in \Sigma_{A, \ell}$ such that $I J \in \Sigma_{A, n+\ell}$ $\left(J I \in \Sigma_{A, n+\ell}\right.$ respectively $)$;

(iii) $\sum_{K: I K J \in \Sigma_{A, i}} s_{i}(I K J, q) \approx s_{n}(I, q) s_{\ell}(J, q) s_{i-n-\ell}(q)$ for all $I \in \Sigma_{A, n}$, $J \in \Sigma_{A, \ell}, i>n+\ell+2 p$.

Proof. For any $I \in \Sigma_{A, \ell}$, write $I=K J$ where $J \in \Sigma_{A, \ell-p}$. By Lemmas 2.1 and 2.2 , we have (note that $p$ is fixed)

$$
s_{\ell}(I, q) \approx s_{\ell-p}(J, q) .
$$

Since $A^{p}>\mathbf{0}$, for $J \in \Sigma_{A, \ell-p}$, we can find $K \in \Sigma_{A, p}$ such that $I=K J \in \Sigma_{A, \ell}$. Hence when we take the sum of $I \in \Sigma_{A, \ell}$ on the left side of the expression, it is $\approx$ to the right side summing over all $J \in \Sigma_{A, \ell-p}$. This implies (i).

To prove (ii), we fix $I \in \Sigma_{A, n}$ and take $J \in \Sigma_{A, \ell}$ such that $I J \in \Sigma_{A, n+\ell}$. By Lemmas 2.2 and 2.1, we have

$$
s_{n+\ell}(I J, q) \approx s_{n}(I, q) s_{\ell}(J, q) .
$$


Thus

$$
\sum_{J} s_{n+\ell}(I J, q) \preccurlyeq s_{n}(I, q) s_{\ell}(q) .
$$

For the reverse inequality we note for any $J^{\prime} \in \Sigma_{A, \ell-p}$, there is $K \in \Sigma_{A, p}$ such that $I K J^{\prime} \in \Sigma_{A, n+\ell}$ and

$$
s_{n+\ell}\left(I K J^{\prime}\right) \approx s_{n}(I, q) s_{p}(K, q) s_{\ell-p}\left(J^{\prime}, q\right) \approx s_{n}(I, q) s_{\ell-p}\left(J^{\prime}, q\right) .
$$

Therefore summing over the above $J^{\prime}$, we have

$$
\sum_{I J \in \Sigma_{A, n+\ell}} s_{n+\ell}(I J, q) \succcurlyeq \sum_{J^{\prime}} s_{n+\ell}\left(I K J^{\prime}, q\right) \approx s_{n}(I, q) s_{\ell-p}(q) \approx s_{n}(I, q) s_{\ell}(q)
$$

(we make used of $A^{p}>\mathbf{0}$ as in (i)). This proves one of the $\approx$ in (ii). The remaining part follows from the same argument.

To prove (iii), we first observe that

$$
\begin{aligned}
\sum_{K: I K J \in \Sigma_{A, i}} s_{i}(I K J, q) & \approx \sum_{K: I K J \in \Sigma_{A, i}} s_{n}(I, q) s_{i-n-\ell}(K, q) s_{\ell}(J, q) \\
& \preccurlyeq s_{n}(I, q) s_{\ell}(J, q) s_{i-n-\ell}(q)
\end{aligned}
$$

On the other hand, for any $K_{1} \in \Sigma_{A, i-n-\ell-2 p}$, there exists $K_{2}, K_{3} \in \Sigma_{A, p}$ such that $I K_{2} K_{1} K_{3} J \in \Sigma_{A, i}$. Therefore

$$
\begin{aligned}
\sum_{K: I K J \in \Sigma_{A, i}} s_{i}(I K J, q) & \succcurlyeq s_{n}(I, q) s_{\ell}(J, q) \sum_{K_{1} \in \Sigma_{A, i-n-\ell-2 p}} s_{i-n-\ell-2 p}\left(K_{1}, q\right) \\
& \approx s_{n}(I, q) s_{\ell}(J, q) s_{i-n-\ell-2 p}(q) \\
& \approx s_{n}(I, q) s_{\ell}(J, q) s_{i-n-\ell}(q) .
\end{aligned}
$$

Lemma 2.4. For a fixed $q \in \mathbb{R}$,

(i) $s_{\ell+n}(q) \approx s_{\ell}(q) s_{n}(q)$.

(ii) $s_{n}(q) \approx \exp (n P(q))$ where $P(q)$ is the pressure function defined in (1.1).

Proof. From Lemma 2.3 (ii), there exist $C, C^{\prime}>0$ such that

$$
C^{\prime} s_{\ell}(q) s_{n}(q) \leq s_{\ell+n}(q) \leq C s_{\ell}(q) s_{n}(q)
$$

which proves (i). To prove (ii), we can write $C s_{\ell+n}(q) \leq\left(C s_{\ell}(q)\right)\left(C s_{n}(q)\right)$. Hence the subadditivity property implies

$$
P(q)=\lim _{n \rightarrow \infty} \frac{\log \left(C s_{n}(q)\right)}{n}=\inf _{n} \frac{\log \left(C s_{n}(q)\right)}{n},
$$

so that $C^{-1} \exp (n P(q)) \leq s_{n}(q)$. The reverse inequality follows from a similar argument.

For each integer $n>0$, let $\mathcal{B}_{n}$ be the $\sigma$-algebra generated by the cylinders $[I], I \in \Sigma_{A, n}$. We define a sequence of probability measures $\left\{\nu_{n, q}\right\}$ on $\mathcal{B}_{n}$ by

$$
\nu_{n, q}([I])=\frac{s_{n}(I, q)}{s_{n}(q)} \quad \forall I \in \Sigma_{A, n} .
$$


Then there is a subsequence $\left\{\nu_{n_{k}, q}\right\}_{k \geq 1}$ converges in the weak-star topology to a probability measure $\nu_{q}$. The following assertion shows that $\nu_{q}$ has the "Gibbs property".

Lemma 2.5. For a fixed $q \in \mathbb{R}, \nu_{q}([I]) \approx s_{n}(I, q) \exp (-n P(q))$ for all $n>$ $0, I \in \Sigma_{A, n}$.

Proof. Let $p$ be such that $A^{p}>\mathbf{0}$. For any $I \in \Sigma_{A, n}$ and $\ell>n+p$, we have

$$
\begin{array}{rlrl}
\nu_{\ell, q}([I]) & =\sum_{J: I J \in \Sigma_{A, \ell}} \nu_{\ell, q}([I J])=\sum_{J: I J \in \Sigma_{A, \ell}} \frac{s_{\ell}(I J, q)}{s_{\ell}(q)} \\
& \approx s_{n}(I, q) \frac{s_{\ell-n}(q)}{s_{\ell}(q)} & \quad \text { (by Lemma 2.3 (i })) \\
& \approx s_{n}(I, q) \exp (-n P(q)) . & \text { (by Lemma 2.4). }
\end{array}
$$

Letting $\ell=n_{k} \uparrow \infty$, we obtain the desired result.

Proof of Theorem 1.1. Fix $q \in \mathbb{R}$. Let $\mu_{q}$ be a limit point of the subsequence of $\left\{\frac{1}{n}\left(\nu_{q}+\nu_{q} \circ \sigma^{-1}+\ldots+\nu_{q} \circ \sigma^{-(n-1)}\right)\right\}$ in the weak-star topology. Then $\mu_{q}$ is a $\sigma$-invariant measure on $\Sigma_{A}$. We have for each $I \in \Sigma_{A, n}$ and $\ell>p$,

$$
\begin{array}{rlr}
\nu_{q} \circ \sigma^{-\ell}([I]) & =\sum_{J: J I \in \Sigma_{A, n+\ell}} \nu_{q}([J I]) \\
& \approx \sum_{J: J I \in \Sigma_{A, n+\ell}} s_{n+\ell}(J I, q) \exp (-(n+\ell) P(q)) \quad \text { (by Lemma 2.5) } \\
& \approx s_{\ell}(q) s_{n}(I, q) \exp (-(\ell+n) P(q)) \quad \text { (by Lemma 2.3 (ii)) } \\
(2.4) & \approx s_{n}(I, q) \exp (-n P(q)) \quad \text { (by Lemma 2.4). }
\end{array}
$$

This proves that $\mu_{q}$ is a Gibbs measure. In what follows we prove that $\mu_{q}$ is ergodic. First we show that there is a constant $C>0$ such that for each $I \in \Sigma_{n}$, $J \in \Sigma_{\ell}$,

$$
\lim _{k \rightarrow \infty} \frac{1}{k} \sum_{i=0}^{k-1} \mu_{q}\left([I] \cap \sigma^{-i}([J])\right) \geq C \mu_{q}([I]) \mu_{q}([J]) .
$$

Since $\mu_{q}$ is supported on $\Sigma_{A}$, it suffices to prove (2.5) for $I \in \Sigma_{A, n}$ and $J \in \Sigma_{A, \ell}$. Note that when $i>n+2 p$,

$$
\begin{aligned}
& \mu_{q}\left([I] \cap \sigma^{-i}([J])\right) \\
= & \sum_{K: I K J \in \Sigma_{A, i+\ell}} \mu_{q}([I K J]) \\
\succcurlyeq & \sum_{K: I K J \in \Sigma_{A, i+\ell}} s_{i+\ell}(I K J, q) \exp (-(i+\ell) P(q)) \\
\approx & s_{n}(I, q) s_{\ell}(J, q) s_{i-n}(q) \exp (-(i+\ell) P(q)) \\
\approx & s_{n}(I, q) s_{\ell}(J, q) \exp (-(n+\ell) P(q)) \\
\approx & \mu_{q}([I]) \mu_{q}([J])
\end{aligned}
$$


from which (2.5) follows. Since the collection $\left\{[I]: I \in \Sigma_{n}, n \in \mathbb{N}\right\}$ is a semialgebra that generates the Borel $\sigma$-algebra on $\Sigma$, a standard argument (e.g., see the proof of [W, Theorem 1.17]) shows that for any Borel sets $A, B \subset \Sigma$,

$$
\lim _{k \rightarrow \infty} \frac{1}{k} \sum_{i=0}^{k-1} \mu_{q}\left(A \cap \sigma^{-i}(B)\right) \geq C \mu_{q}(A) \mu_{q}(B) .
$$

This implies that for any Borel sets $A, B \subset \Sigma$ with $\mu_{q}(A)>0, \mu_{q}(B)>0$, there exists $n>0$ with $\mu_{q}\left(A \cap \sigma^{-n}(B)\right)>0$. By [W, Theorem 1.5], $\mu_{q}$ is ergodic.

For the uniqueness we recall that any two distinct ergodic measures must be singular to each other; but the Gibbs property (1.2) implies that any two $\mu_{q}$ must be absolutely continuous to each other. Hence $\mu_{q}$ must be unique.

Corollary 2.6. Let $\mu_{q}$ be the Gibbs measure in Theorem 1.1. There exists $C>$ 0 such that for any $I \in \Sigma_{A, n}, J \in \Sigma_{A, \ell}$ with $I J \in \Sigma_{A, n+\ell}$,

$$
C^{-1} \mu_{q}([I J]) \leq \mu_{q}([I]) \mu_{q}([J]) \leq C \mu_{q}([I]) \mu_{q}([J]) .
$$

Proof. We have seen from the proof of Lemma 2.3 that for the above $I, J$, $s_{n+\ell}(I J, q) \approx s_{n}(I, q) s_{\ell}(J, q)$ and from Lemma 2.4, $s_{\ell+n}(q) \approx s_{n}(q) s_{\ell}(q)$. By the definition of $\nu_{n, q}$, we have

$$
\nu_{n, q}([I J]) \approx \nu_{n, q}([I]) \nu_{n, q}([J])
$$

which implies that the Gibbs measure $\mu_{q}$ has the same property.

The above property is called quasi-Bernoulli property by Heurteaux [H] (we remark that Heurteaux only introduced and studied it for measures in the full shift space $\Sigma$ ). To prove Theorem 1.2 , we need a result in $[\mathrm{H}]$. Let $\eta$ be a probability measure on $\Sigma$. For $q \in \mathbb{R}$, let $\tau_{\eta}(q)$ be the $L^{q}$-spectrum of $\eta$, i.e.,

$$
\tau_{\eta}(q)=\liminf _{n \rightarrow \infty} \frac{\log \sum_{I} \eta([I])^{q}}{\log m^{-n}}
$$

where the summation is taken over all $I \in \Sigma_{n}$ with $\eta([I]) \neq 0$.

Proposition 2.7. ([H, Theorem 2.1]) Let $\eta$ be a probability measure on $\Sigma$. Assume that there exists a constant $C>0$ such that

$$
\eta([I J]) \leq C \eta([I]) \eta([J]) \quad \forall I \in \Sigma_{n}, J \in \Sigma_{\ell} .
$$

Then $\tau_{\eta}^{\prime}(1)$ exists if $\eta$ is a Young measure (i.e., $\lim _{n \rightarrow \infty} \frac{\log \eta\left(I_{n}(x)\right)}{\log m^{-n}}=$ constant for $\eta$ almost all $x=\left(j_{i}\right) \in \Sigma$, here $\left.I_{n}(x)=\left[j_{1} \ldots j_{n}\right]\right)$. This is the case when $\eta$ is quasi-Bernoulli.

Proof of Theorem 1.2. For each $q$, let $\mu_{q}$ be the corresponding Gibbs measure in Theorem 1.1. We can view $\mu_{q}$ as a measure on $\Sigma$. For $t \in \mathbb{R}$, let $\tau_{\mu_{q}}(t)$ be the 
$L^{t}$-spectrum of $\mu_{q}$. Since $\mu_{q}$ has the Gibbs property, it is easy to show by the definition of $L^{t}$-spectrum that

$$
\tau_{\mu_{q}}(t)=\frac{t P(q)-P(q t)}{\log m} .
$$

Note that $\mu_{q}$ satisfies the condition (2.6). Since $\mu_{q}$ is ergodic on $\Sigma$, it is a Young measure by the Shannon-McMillan-Brieman theorem (i.e., $\lim _{n \rightarrow \infty} \frac{-\log \mu_{q}\left(I_{n}(x)\right)}{n}$ equals the entropy of $\mu_{q}$ (with respect to $\sigma$ ) for $\mu_{q}$-almost all $x=\left(j_{i}\right) \in \Sigma$ and $\left.I_{n}(x)=\left[j_{1} \ldots j_{n}\right]\right)$. Hence by Proposition 2.7, $\tau_{\mu_{q}}(t)$ is differentiable at $t=1$. This implies that $P(q)$ is differential at any fixed $q \neq 0$, and

$$
P^{\prime}(q)=\frac{P(q)-\log m \cdot \tau_{\mu_{q}}^{\prime}(1)}{q}
$$

Proof of Theorem 1.3. Let $\alpha=\tau^{\prime}(q)$ with $q \neq 0$. Let $\mu_{q}$ be the corresponding Gibbs measures in Theorem 1.1, then (2.7) implies that

$$
\tau_{\mu_{1}}(q)=\frac{q P(1)-P(q)}{\log m}
$$

and

$$
E(\alpha)=\left\{x \in \Sigma: \lim _{n \rightarrow \infty} \frac{\log \mu_{1}\left(\left[x_{1} \cdots x_{n}\right]\right)}{\log m^{-n}}=\frac{P(1)-\alpha}{\log m}\right\}
$$

By [BMP, Theorem 1] or [LN2, Theorem 4.1], we have

$$
\operatorname{dim}_{H} E(\alpha) \leq\left(\frac{P(1)-\alpha}{\log m}\right) q-\tau_{\mu_{1}}(q)=\frac{1}{\log m}(-\alpha q+P(q)) \quad \forall q \in \mathbb{R} .
$$

For the reverse inequality, we see from the proof of Theorem 1.2 that $\tau_{\mu_{q}}^{\prime}(1)$ exists and

$$
\tau_{\mu_{q}}^{\prime}(1)=\frac{-q P^{\prime}(q)+P(q)}{\log m} .
$$

By $[\mathrm{N}]$, we have for $\mu_{q}$ almost all $I=\left(i_{1} \ldots i_{n} \ldots\right) \in \Sigma$,

$$
\lim _{n \rightarrow \infty} \frac{\log \mu_{q}\left(\left[i_{1}, \ldots, i_{n}\right]\right)}{\log m^{-n}}=\tau_{\mu_{q}}^{\prime}(1)=\frac{-q P^{\prime}(q)+P(q)}{\log m} .
$$

This implies that

$$
\lim _{n \rightarrow \infty} \frac{\log \left\|M(x) M(\sigma x) \cdots M\left(\sigma^{n-1} x\right)\right\|}{n}=P^{\prime}(q)=\alpha \quad \mu_{q}-\text { a.a. } x \in \Sigma .
$$

Therefore we have

$$
\operatorname{dim}_{H} E(\alpha) \geq \operatorname{dim}_{H} \mu_{q}=\frac{-q \alpha+P(q)}{\log m} .
$$




\section{Nonnegative matrices}

In this section, we always assume that $M$ is a function on $\Sigma_{A}$ taking values in the set of all $d \times d$ non-negative matrices and satisfies (H1) and (H2) defined in Section 1. Let $q>0$ be fixed. Then $s_{n}(I, q)$ and $s_{n}(q)$ in (2.1) are reduced to

$$
s_{n}(I, q)=\left\|M_{I}\right\|^{q} \quad \forall I \in \Sigma_{A, n} \quad \text { and } \quad s_{n}(q)=\sum_{I \in \Sigma_{A, n}} s_{n}(I, q) .
$$

For convenience, we let

$$
b=\min _{s, t \in\{1,2, \ldots m\}} \min _{1 \leq i, j \leq d}\left(\sum_{k=1}^{r} \sum_{K \in \Sigma_{A, k ; s, t}} M_{K}\right)_{i, j} .
$$

Then $b>0$ by (H2).

We will reformulate the three theorems in the previous section. The proofs are almost the same and for simplicity, we only point out the differences. Here Lemmas 2.1, 2.2 do not hold anymore; on the other hand we can use (H2) to replace these lemmas to obtain an analog of Lemma 2.3:

Lemma 3.1. For a fixed $q>0$,

(i) $s_{\ell+1}(q) \approx s_{\ell}(q)$.

(ii) For $I \in \Sigma_{A, n}, \sum_{J} s_{n+\ell}(I J, q) \approx \sum_{J} s_{n+\ell}(J I, q) \approx s_{n}(I, q) s_{\ell}(q)$ where the first (second) sum is taken over all $J \in \Sigma_{A, \ell}$ such that IJ $\in \Sigma_{A, n+\ell}$ ( $J I \in \Sigma_{A, n+\ell}$ respectively).

(iii) $\sum_{k=1}^{2 r} \sum_{K: I K J \in \Sigma_{A, i+k}} s_{i+k}(I K J, q) \approx s_{n}(I, q) s_{\ell}(J, q) s_{i-n-\ell}(q)$ for all $I \in$ $\Sigma_{A, n}, \quad J \in \Sigma_{A, \ell}, i>n+\ell$.

Proof. For any $I \in \Sigma_{A, \ell+1}$, write $I=i J$ with $J \in \Sigma_{A, \ell}$. Using $\left\|M_{I}\right\| \leq$ $\left\|M_{i}\right\|\left\|M_{J}\right\|$, we have for $q>0$,

$$
s_{\ell+1}(q) \leq m\left(\sup _{i \in\{1,2, \ldots, m\}}\left\|M_{i}\right\|^{q}\right) s_{\ell}(q) .
$$

That is, $s_{\ell+1}(q) \preccurlyeq s_{\ell}(q)$. For the reverse inequality, since for any $J \in \Sigma_{A, \ell}$,

$$
\sum_{k=1}^{r} \sum_{K \in \Sigma_{A, k}: K J \in \Sigma_{A, \ell+k}}\left\|M_{K J}\right\|=\left\|\left(\sum_{k=1}^{r} \sum_{K \in \Sigma_{A, k}: K J \in \Sigma_{A, \ell+k}} M_{K}\right) M_{J}\right\| \geq b\left\|M_{J}\right\|,
$$

it follows that

$$
\sum_{k=1}^{r} \sum_{K \in \Sigma_{A, k}: K J \in \Sigma_{A, \ell+k}}\left\|M_{K J}\right\|^{q} \geq\left(\frac{b}{\sum_{k=1}^{r} m^{k}}\right)^{q}\left\|M_{J}\right\|^{q} .
$$

This combines with $s_{\ell+1}(q) \preccurlyeq s_{\ell}(q)$ imply that

$$
s_{\ell+1}(q) \succcurlyeq \sum_{k=1}^{r} s_{\ell+k}(q) \geq s_{\ell}(q)
$$

and completes the proof of (i). 
To prove (ii), it follows from the inequality $\left\|M_{I J}\right\| \leq\left\|M_{I}\right\|\left\|M_{J}\right\|$ that $\sum_{J \in \Sigma_{A, \ell}: I J \in \Sigma_{A, n+\ell}} s_{n+\ell}(I J, q) \preccurlyeq s_{n}(I, q) s_{\ell}(q)$. For the reverse inequality, we use (H2) as above to conclude that for any $J \in \Sigma_{A, \ell}$,

$$
\sum_{k=1}^{r} \sum_{K \in \Sigma_{A, k}: I K J \in \Sigma_{A, n+\ell+k}}\left\|M_{I K J}\right\| \succcurlyeq\left\|M_{I}\right\|\left\|M_{J}\right\| .
$$

Hence $\sum_{k=1}^{r} \sum_{K \in \Sigma_{A, k}: I K J \in \Sigma_{A, n+\ell+k}}\left\|M_{I K J}\right\|^{q} \succcurlyeq\left\|M_{I}\right\|^{q}\left\|M_{J}\right\|^{q}$ and therefore, summing over the $J \in \Sigma_{A, \ell}$,

$$
\sum_{k=1}^{r} \sum_{L \in \Sigma_{A, \ell+k}: I L \in \Sigma_{A, n+\ell+k}} s_{n+\ell+k}(I L, q) \succcurlyeq s_{n}(I, q) s_{\ell}(q) .
$$

Since

we have

$$
\sum_{J \in \Sigma_{A, \ell}: I J \in \Sigma_{A, n+\ell}} s_{n+\ell}(I J, q) \succcurlyeq \sum_{J \in \Sigma_{A, \ell+1}: I J \in \Sigma_{A, n+\ell+1}} s_{n+\ell+1}(I J, q)
$$

$$
\sum_{J \in \Sigma_{A, \ell}: I J \in \Sigma_{A, n+\ell}} s_{n+\ell}(I J, q) \succcurlyeq \sum_{k=1}^{r} \sum_{J \in \Sigma_{A, \ell+k}: I J \in \Sigma_{A, n+\ell+k}} s_{n+\ell+k}(I J, q) \succcurlyeq s_{n}(I, q) s_{\ell}(q) .
$$

This completes the proof of an $\approx$ in (ii); the other $\approx$ follows from an identical argument.

To prove (iii), we have,

$$
\begin{aligned}
\sum_{k=1}^{2 r} \sum_{K: I K J \in \Sigma_{A, i+k}} s_{i+k}(I K J, q) & \leq s_{n}(I, q) s_{\ell}(J, q) \sum_{k=1}^{2 r} s_{i+k-n-\ell}(q) \\
& \approx s_{n}(q) s_{\ell}(J, q) s_{i-n-\ell}(q) \quad(\text { by }(i)) .
\end{aligned}
$$

On the other hand, for any $W \in \Sigma_{A, i-n-\ell}$, by (H2), there exist $1 \leq k_{1} \leq r$, $K_{1} \in \Sigma_{A, k_{1}}$ such that $I K_{1} W \in \Sigma_{A, i-\ell+k_{1}}$ and

$$
\left\|I K_{1} W\right\| \geq \frac{b\|I\|\|W\|}{\sum_{k=1}^{r} m^{k}}
$$

where $b$ is defined by (3.1). By using (H2) again, there exist $1 \leq k_{2} \leq r$, $K_{2} \in \Sigma_{A, k_{2}}$ such that $I K_{1} W K_{2} J \in \Sigma_{A, i+k_{1}+k_{2}}$ and

$$
\left\|I K_{1} W K_{2} J\right\| \geq \frac{b\left\|I K_{1} W\right\|\|J\|}{\sum_{k=1}^{r} m^{k}} \geq \frac{b^{2}\|I\|\|W\|\|J\|}{\left(\sum_{k=1}^{r} m^{k}\right)^{2}} .
$$

Therefore we have

$$
\sum_{k=1}^{2 r} \sum_{K: I K J \in \Sigma_{A, i+k}} s_{i+k}(I K J, q) \succcurlyeq s_{n}(I, q) s_{\ell}(J, q) s_{i-n-\ell}(q)
$$

and (iii) follows.

We now state the corresponding theorems as in Section 1. 
Theorem 3.2. Suppose $M$ is a function on $\Sigma_{A}$ taking values in the set of all $d \times d$ non-negative matrices and satisfies (H1) and (H2). Then for any $q>0$, there is a unique Gibbs measure $\mu_{q}$ on $\Sigma_{A}$ as in Theorem 1.1.

The proof is almost identical to that of Theorem 1.1, using Lemma 3.1. The only adjustment is to replace

$$
\mu_{q}\left([I] \cap \sigma^{-i}([J])\right) \approx \mu_{q}(I) \mu_{q}(J), \quad i>n+2 p .
$$

by

$$
\sum_{i=1}^{2 r} \mu_{q}\left([I] \cap \sigma^{-i}([J])\right) \approx \mu_{q}(I) \mu_{q}(J) \quad \forall i>n .
$$

We use the same proof as in Section 2 for the next two theorems.

Theorem 3.3. Under the same conditions of Theorem 3.2, $P(q)$ is differentiable for any $q>0$.

Theorem 3.4. Under the same conditions of Theorem 3.2, we have for any $\alpha=P^{\prime}(q), q>0$,

$$
\operatorname{dim}_{H} E(\alpha)=\frac{1}{\log m}(-\alpha q+P(q))
$$

where $E(\alpha)=\left\{J=\left(j_{i}\right) \in \Sigma_{A}: \lim _{n \rightarrow \infty} \log \left\|M_{j_{1}} \cdots M_{j_{n}}\right\| / n=\alpha\right\}$.

To relate Theorem 3.4 to the classical random product of matrices, we let $\left\{Y_{n}\right\}$ be the i.i.d. random variables that take values $M_{1}, \ldots, M_{m}$, invertible matrices and with uniform distribution, then $\lim _{n \rightarrow \infty} \frac{1}{n} \log \left\|Y_{n} \ldots Y_{1}\right\|=\lambda$ a.s. and $\lambda$ is called the upper Lyapunov exponent ([FK], [BL, Chapter 1]). In comparison with Theorem 3.4, we let $\Sigma_{A}=\Sigma$ be the space of full shift (i.e., all the entries of $A$ are 1$)$, then $P(0)=\log m$. The limit of the random variables corresponds to the case for $q=0, \lambda=P^{\prime}(0)$ and $\operatorname{dim}_{H} E(\lambda)=P(0) / \log m=1$ (the existence of the derivative follows from some additional assumptions on the $M_{j}$ ([BL, p.119]).

We remark that if $\Sigma_{A}=\Sigma$, then condition (H2) is reduced to a more simple form: $\sum_{k=1}^{r} H^{k}>\mathbf{0}$ where $H=M_{1}+\cdots+M_{m}$. The condition is essential for the theorems in Section 3. Indeed we have:

Example 3.5. Let $M_{1}=\left(\begin{array}{cc}2 & 0 \\ 0 & 1\end{array}\right)$ and $M_{2}=\left(\begin{array}{cc}2 & 0 \\ 0 & 3\end{array}\right)$. Then $H=M_{1}+M_{2}$ is reducible. Since $M_{J}=\left(\begin{array}{ll}2^{n} & 0 \\ 0 & 3^{k}\end{array}\right)$ where $|J|=n$ and $k$ is the number of 2 's appeared in $J \cdot \sum_{|J|=n}\left\|M_{J}\right\|^{q}=\sum_{k=0}^{n}\left(\begin{array}{l}n \\ k\end{array}\right)\left(2^{n}+3^{k}\right)^{q}$. Note that

$\sum_{k=0}^{n}\left(\begin{array}{l}n \\ k\end{array}\right)\left(2^{n}+3^{k}\right)^{q} \geq \max \left\{\sum_{k=0}^{n}\left(\begin{array}{l}n \\ k\end{array}\right) 2^{n q}, \sum_{k=0}^{n}\left(\begin{array}{l}n \\ k\end{array}\right) 3^{k q}\right\}=\max \left\{2^{n(q+1)},\left(1+3^{q}\right)^{n}\right\}$

and

$$
\sum_{k=0}^{n}\left(\begin{array}{l}
n \\
k
\end{array}\right)\left(2^{n}+3^{k}\right)^{q} \leq \sum_{k=0}^{n}\left(\begin{array}{l}
n \\
k
\end{array}\right) 2^{q}\left(2^{n q}+3^{k q}\right)=2^{q}\left(2^{n(q+1)}+\left(1+3^{q}\right)^{n}\right) .
$$


We have $P(q)=\max \left\{(q+1) \log 2, \log \left(1+3^{q}\right)\right\}$, which is not differentiable at $q=1$.

We see that the Gibbs measure $\mu_{q}$ in Section 2 has the quasi-Bernoulli property. However for the case of non-negative matrices, only $\mu_{q}([I J]) \leq$ $C \mu_{q}([I]) \mu_{q}([J]), I \in \Sigma_{n}, J \in \Sigma_{\ell}$. The following example shows that the reverse inequality may not hold.

Example 3.6. Let $M_{1}=\left(\begin{array}{ll}1 & 1 \\ 0 & 1\end{array}\right)$ and $M_{2}$ an arbitrarily positive matrix, then $H=M_{1}+M_{2}$ is an irreducible positive matrix. Let $J=1 \ldots 1$ ( $n$-times), then $\left\|M_{J}\right\|=n+2$ and hence

$$
\left\|M_{J}\right\|\left\|M_{J}\right\| \geq \frac{n}{2}\left\|M_{J J}\right\| .
$$

Since $\mu_{q}(I) \approx \exp (-n P(q)) \cdot\left\|M_{I}\right\|, I \in \Sigma_{n}$, we see that there does not exist $C^{\prime}>0$ such that $C^{\prime} \mu_{q}([J]) \mu([J]) \leq \mu([J J])$.

\section{Examples and remarks}

Consider the classical Bernoulli convolution $X=(1-\rho)^{-1} \sum_{n=1}^{\infty} \rho^{n} X_{n}$ where the $X_{n}$ 's are i.i.d. random variables which take values 0,1 and with probability $1 / 2$ on each value. Let $\mu_{\rho}$ be the distribution measure of $X$. It is well known that for $0<\rho<1 / 2$, the measure is a Cantor type measure. It was proved recently that $\mu_{\rho}$ is absolutely continuous for almost all $1 / 2<\rho<1$ [S], however, it is still not clear which $\mu_{\rho}$ is absolutely continuous or singular. The question has been subjected to intensive investigation. The reader can refer to the survey articles $[\mathrm{L}],[\mathrm{PSS}]$ and the references there. The interest of the Bernoulli convolution in our setting is that the $\mu_{\rho}$ satisfies the self-similar identity

$$
\mu_{\rho}=\frac{1}{2} \mu_{\rho} S_{1}^{-1}+\frac{1}{2} \mu_{\rho} S_{2}^{-1}
$$

where $S_{1} x=\rho x, S_{2} x=\rho x+(1-\rho) ;\left\{S_{1}, S_{2}\right\}$ is the iterated function system (IFS). The support of $\mu_{q}$ is $[0,1]$. For $0<\rho<1 / 2$, the $S_{J}(0,1)$ 's are disjoint (as in the basic intervals of the Cantor set); for $1 / 2<\rho<1$, the $S_{J}(0,1)$ overlaps which is the source of difficulty.

For $\rho=(\sqrt{5}-1) / 2$, the reciprocal of the golden ratio, it was shown by Erdös that $\mu_{\rho}$ is singular. In order to consider the multifractal structure of $\mu_{\rho}$, we can put the IFS $\left\{S_{1}, S_{2}\right\}$ to a new set of IFS $\left\{R_{i}\right\}_{i=1}^{3}$ with no overlap:

$$
R_{1}(x)=\rho^{2} x, \quad R_{2}(x)=\rho^{3} x+\rho^{2}, \quad \text { and } \quad R_{3}(x)=\rho^{2} x+\rho .
$$

Then the measure $\mu_{\rho}$ satisfies

$$
\mu_{\rho}\left(\left[i_{1} \ldots i_{n}\right]\right) \approx \frac{1}{4^{n}}\left\|M_{i_{1}} \ldots M_{i_{n}}\right\|
$$

where $\left[i_{1} \ldots i_{n}\right]=R_{i_{1}} \ldots R_{i_{n}}([0,1])$ and

$$
M_{1}=\left(\begin{array}{cc}
1 & 1 \\
0 & 1
\end{array}\right), \quad M_{2}=\left(\begin{array}{cc}
\frac{1}{2} & \frac{1}{2} \\
\frac{1}{2} & \frac{1}{2}
\end{array}\right) \quad \text { and } \quad M_{3}=\left(\begin{array}{cc}
1 & 0 \\
1 & 1
\end{array}\right)
$$


([LN1], [Fe1],[FeO]). The $\left\{M_{i}\right\}_{i=1}^{3}$ satisfies the conditions (H2). Hence by Theorem 3.2, 3.3, $P(q)$ is differentiable for $q>0$ and the multifractal formalism holds.

Actually, more can be said about the $L^{q}$-spectrum $\tau(q)$ of $\mu_{q}$ : an explicit formula was given in [LN1] for $q>0$ and was extended to $q<0$ in [Fe1]. By using the formula it was proved that $\tau(q)$ is differentiable (actually real analytic) on $\mathbb{R}$ except one point in $\mathbb{R}^{-}$in [Fe1].

The above example of Bernoulli convolution gives rise to another interesting question. Note that the above example is a special case of the overlapping IFS that can be reduced to new sets of IFS with no overlap and the calculation of the $\tau(q)$ can be converted into the product of matrices. Such IFS forms an important subclass of those that satisfy the weak separation condition ([LN2], $[\mathrm{LNR}]$ )(it will be interesting to classify this subclass of IFS). Under the weak separation condition it was proved that the multifractal formalism is valid provided that $\tau(q)$ is differentiable [LN2]. However we do not know its differentiability in the general case. In a forthcoming paper [Fe2], the first author proves the differentiability of $\tau(q)$ for $q>0$ in the case that the IFS is equicontractive and satisfies the finite type condition (see $[\mathrm{NW}]$ ).

The behavior for $q<0$ is also important for the multifractal analysis. There is no problem when $M$ is a positive matrix-valued function as we considered in Section 2. For the non-negative matrix-valued $M, M_{J}$ can be $\mathbf{0}$, we have to modify the pressure function $P(q)$ in (1.6) slightly:

$$
P(q)=\lim _{n \rightarrow \infty} \frac{1}{n} \log \sum_{J \in \mathcal{N}_{n}}\left\|M_{J}\right\|^{q}
$$

where $\mathcal{N}_{n}$ consists of all the $J \in \Sigma_{A, n}$ such that $M_{J} \neq \mathbf{0}$. It is clear that if $M_{J} \neq \mathbf{0}$ for all $J \in \Sigma_{A, n}$, then the super-additivity of the sum in (4.1) implies that the limit exists. We include a simple proposition with $\Sigma_{A}=\Sigma$ to set up the consideration:

Proposition 4.4 Suppose $M_{1}, \cdots, M_{m}$ are non-negative matrices and $H=$ $\sum_{i=1}^{m} M_{i}$ is irreducible, then the limit in (4.1) exists for each $q<0$.

Proof. By the irreducibility, there exists integer $r$ with $\sum_{k=1}^{r} H^{k}>0$. Hence there is a constant $C>0$ such that for any two finite indices $I, J$, there exists $K_{0} \in \bigcup_{k=1}^{r} \Sigma_{k}$ satisfying

$$
0<\left\|M_{I K_{0} J}\right\| \leq C\left\|M_{I}\right\|\left\|M_{J}\right\|
$$

Denote by $s_{n}=\sum_{J \in \mathcal{N}_{n}}\left\|M_{J}\right\|^{q}$. Then (4.2) implies $s_{n} s_{\ell} \leq C^{-q} \sum_{k=1}^{r} s_{n+\ell+k}$. From (4.2) we also deduce that for any finite index $I$, there exists $i \in \Sigma_{1}$ such that $M_{I i} \neq 0$; Since $\left\|M_{I i}\right\| \leq C_{1}\left\|M_{I}\right\|$ for some constant $C_{1}>0$, we have $s_{n} \leq C_{1}^{-q} s_{n+1}$ for any integer $n, \ell$. It follows that $s_{n} s_{\ell} \leq C^{\prime} s_{n+\ell+r}$ for some constant $C^{\prime}>0$ (depending on $q$ ), which implies that $a_{n}=\frac{1}{C^{\prime}} s_{n-r}$ is supermultiplicative. This yields the existence of the limit. 
The differentiability of such $P(q)$ for $q<0$ is still unknown. We know that in the above Bernoulli convolution of the golden ratio, it is possible for the $P(q)$ to be non-differentiable at a point of $q<0$ [Fe1]. On the other hand, it is known that by imposing some stronger conditions on the matrices, the pressure function $P(q)$ is analytic near $q=0$ (see e.g., [BL, Theorem 4.3]).

Finally we remark that we do not know whether the theorems can be extended to matrices with entries in $\mathbb{R}$. An important theorem concerning this is in [BL, Theorem 4.3] for the analyticity of $P(q)$ near zero. Much closer to our development is the scaling functions: $f(x)=\sum_{i=0}^{m} c_{i} f(2 x-i)$. It is known that such function can be put into matrix form as in the previous example [DL1]. Daubechies and Lagarias studied the multifractal formalism of the well known scaling function $D_{4}$ [DL2]. They showed the differentiability of the corresponding $\tau(q)$, but the consideration depends on the two $2 \times 2$ matrices involved to have a common eigenvector. There are some extensions in [LM].

\section{Acknowledgment}

The paper was originally written for the random products of $m$ matrices. The authors would like to thank the referee for the suggestion to modify it to the present form which can be appealed to more general situation. They also thank E. Olivier for introducing the multifractal results of $[\mathrm{BMP}, \mathrm{H}]$ and $\ddot{O}$. Stenflo for reading the manuscript carefully and suggesting some improvements.

\section{References}

[B] R. Bowen, Equilibrium states and the ergodic theory of Anosov diffeomorphisms, Lecture notes in Math., Vol. 470. Springer-Verlag, Berlin-New York, 1975.

[BMP] G. Brown, G. Michon and J. Peyrière, On the multifractal analysis of measures, J. Statist. Phys. 66 (1992), no. 3-4, 775-790.

[BL] P. Bougerol and J. Lacroix, Products of random matrices with applications to Schödinger operators, Progress in Probability and Statistics, 8. Birkhäuser Boston, Inc., Boston, MA, 1985.

[C] L. Carleson, On the support of harmonic for sets of Cantor type, Ann. Acad. Sci. Fenn. Ser. A I Math. 10 (1985), 113-123.

[DL1] I. Daubechies and J. Lagarias, Two-scale difference equations. II. Local regularity, infinite products of matrices and fractals, SIAM J. Math. Anal. 23 (1992), no. 4, 10311079.

[DL2] - On the thermodynamic formalism for multifractal functions, Rev. Math. Phys. 6 (1994), no. 5A, 1033-1070.

[FK] H. Furstenberg and H. Kesten, Products of random matrices, Ann. Math. Statist. 31 (1960), 457-469.

[FL] A. H. Fan and K. S. Lau, Iterated function system and Ruelle operator, J. Math. Anal. Appl. 231 (1999), no. 2, 319-344.

[FLN] A. H. Fan, K.S. Lau and S. M. Ngai, Iterated function systems with overlaps, Asian J. Math. 4 (2000), no. 3, 527-552.

[Fe1] D. J. Feng, The limit Rademacher functions and Bernoulli convolutions associated with Pisot numbers. I,II. preprint.

[Fe2] - The smoothness of $L^{q}$-spectrum of self-similar measures with overlaps, preprint. 
[FeO] D. J. Feng and E. Olivier, Multifractal analysis of the weak Gibbs measures and phase transition - application to some Bernoulli convolutions, preprint.

[H] Y. Heurteaux, Estimations de la dimension inférieure et de la dimension supérieure des mesures, Ann. Inst. H. Poincaré Probab. Statist. 34 (1998), no. 3, 309-338.

[L] K. S. Lau, Iteration function systems with overlaps and multifractal structure, Trends in probability and related analysis (Taipei, 1998), 35-76, World Sci. Publishing, River Edge, NJ, 1999.

[LM] K.S. Lau and M.F. Ma, The regularity of $L^{p}$-scaling functions, Asian J. Math 1 (1997), no. 2, 272-292.

[LN1] K.S. Lau and S.M. Ngai, $L^{q}$-spectrum of the Bernoulli convolution associated with the golden ratio, Studia Math. 131 (1998), no. 3, 225-251.

[LN2] - Multifractal measures and a weak separation condition, Adv. Math. 141 (1999), no. 1, 45-96.

[LNR] K.S. Lau, S. M. Ngai and H. Rao, Iterated function systems with overlaps and selfsimilar measures, J. London Math. Soc. (2) 63 (2001), no. 1, 99-116.

[MU] D. Mauldin and M. Urbanski, Dimension and measures in infinite iterated function systems, Proc. London Math. Soc. (3) 73 (1996), no. 1, 105-154.

[N] S. M. Ngai, A dimension result arising from the $L^{q}$ spectrum of a measure, Proc. Amer. Math. Soc. 125 (1997), no. 10, 2943-2951.

[NW] S. M. Ngai and Y. Wang, Hausdorff dimension of self-similar sets with overlaps, J. London Math. Soc. (2) 63 (2001), no. 3, 655-672.

[PSS] Y. Peres, W. Schlag and B. Solomyak, Sixty years of Bernoulli convolutions, Fractal geometry and stochastics, II (Greifswald/Koserow, 1998), 39-65, Progr. Probab., 46, Birkhäuser, Basel, 2000.

[P] Y. B. Pesin, Dimension theory in dynamical systems, Contemporary views and applications. Chicago Lectures in Mathematics. University of Chicago Press, Chicago, IL, 1997.

[R] D. Ruelle, Thermodynamic formalism. The mathematical structures of classical equilibrium statistical mechanics, Encyclopedia of Mathematics and its Applications, 5. Addison-Wesley Publishing Co., Reading, MA, 1978.

[S] B. Solomyak, On the random series $\sum \pm \lambda^{i}$ (an Erdös problem), Ann. of Math. (2) 142 (1995), no. 3, 611-625.

[W] P. Walters, An introduction to ergodic theory, Graduate Texts in Mathematics, 79. Springer-Verlag, New York-Berlin, 1982.

Department of Mathematical Sciences, Tsinghua University, Beijing, 100084, P.R. China.

Institute of Mathematical Sciences, The Chinese University of Hong Kong, Hong Kong, China.

E-mail address: dfeng@math.tsinghua.edu.cn

Department of Mathematics, the Chinese University of Hong Kong, Hong Kong, CHINA.

E-mail address: kslau@math.cuhk.edu.hk 